\title{
Estudo de série histórica de casos de tuberculose entre 1999 e 2015, em Ouro Preto, Minas Gerais, Brasil
}

\section{Study of historical series of tuberculosis cases between 1999 and 2015, in Ouro Preto, Minas Gerais, Brazil}

Danilo Jorge da Silva' (D), Frederico Prado Abreu (D), Luiz Eduardo de Freitas Xavier ${ }^{1}$ (D), Luana Pimentel Duarte ${ }^{1}$ (D), Vinícius de Jesus Rodrigues Neves (D), Anelise Andrade de Souza ${ }^{2}$ (D), Samuel Ribeiro Dias ${ }^{3}$ (D), Ana Paula Scalia Carneiro ${ }^{4}$ (D), Olivia Maria de Paula Alves Bezerra' (D)

'Universidade Federal de Ouro Preto (UFOP) - Ouro Preto, MG, Brasil

2Instituto René Rachou - Fiocruz Minas, Belo Horizonte, MG, Brasil

${ }^{3}$ Instituto Universitário Italiano de Rosário, Santa Fe, Argentina

${ }^{4}$ Hospital das Clínicas, Universidade Federal de Minas Gerais (UFMG), Belo Horizonte, MG, Brasil
Como citar: Silva DJ, Abreu FP, Xavier LEF, Duarte LP, Neves VJR, Souza AA, et al. Estudo de série histórica de casos de tuberculose entre 1999 e 2015, em Ouro Preto, Minas Gerais, Brasil. Cad Saúde Colet, 2021;29(1):36-45. https://doi.org/10.1590/1414-462X202129010120

\section{Resumo}

Introdução: A tuberculose, caracterizada pela Organização Mundial de Saúde (OMS) como emergência sanitária mundial, é uma doença de impacto global. Objetivo: Realizar série histórica de casos de tuberculose em um período de 17 anos em Ouro Preto, Minas Gerais, considerando a histórica relação da doença com a mineração. Método: Dados foram obtidos em sistema próprio de registros do município, por busca ativa em prontuários médicos e comparados à quantidade de casos notificados no Sistema de Informação de Agravos de Notificação (SINAN). Para análises de tendência, foram utilizados modelos de regressão polinomial para séries históricas. Resultados: Idade média dos casos foi 40,3 $\pm 16,4$ anos. Homens apresentaram 2,23 vezes mais casos e chance 2,07 vezes maior para desfechos negativos. A forma mais observada foi pulmonar (84\%), e sorologia para HIV foi realizada em apenas $16,3 \%$ dos registros. Principal desfecho observado foi cura (70\%), e desfechos negativos totalizaram $20,2 \%$ dos registros. Taxa de incidência média foi 29,76 e 16,23 casos/100 mil habitantes na área municipal e distrital, respectivamente. Conclusão: Apesar da relação histórica entre mineração e tuberculose no município, observa-se que este ainda apresenta preocupantes vulnerabilidades em relação à vigilância da doença. Análise de série temporal sugeriu declínio na proporção de casos curados entre 2009 e 2015.

Palavras-chave: tuberculose; epidemiologia; distribuição geográfica; série histórica.

\begin{abstract}
Background:Tuberculosis, characterized by the World Health Organization as a global health emergency, is a disease of global impact. Objective: To investigate a series of tuberculosis cases during 17 years in Ouro Preto, Minas Gerais, Brazil, considering the historic relationship between mining and the disease. Method: Data was obtained through the city's system of tuberculosis notifications, plus active search through medical records, and compared to the amount of notified cases present in the System of Information of Notifications Complications. For trend analysis, polynomial regression models were used for the historic series. Results: The average age was $40.3 \pm 16.4$ years old. Men showed 2.23 times more cases and chances (odds ratio) of 2.07 times higher for negative outcomes. The most observed form was lung (85\%) and HIV serology was performed in only $16.3 \%$ of the logs. The main observed outcome was a
\end{abstract}

Trabalho realizado em Ouro Preto (MG), Brasil.

Correspondência: Danilo Jorge da Silva. E-mail: danilomachadosilva@gmail.com

Fonte de financiamento: Programa Institucional de Bolsas de Iniciação Científica (PIBIC); Programa de Iniciação à Pesquisa (PIP)

Conflito de interesses: nada a declarar.

Recebido em: Mar. 30, 2019. Aceito em: Jan. 16, 2020
Este é um artigo publicado em acesso aberto (Open Access) sob a licença Creative Commons Attribution, que permite uso, distribuição e reprodução em qualquer meio, sem restrições desde que o trabalho original seja corretamente citado. 
cure (70\%) and negative outcomes accounted for $20.2 \%$ of the logs. The average incidence rate was 29.76 and 16.23 cases/100 thousand inhabitants in the city and district zones, respectively. Conclusion: Despite the historical relationship between mining and tuberculosis in the municipality, it is observed that the same still presents worrying vulnerabilities about disease surveillance, temporal series analysis suggested a decline in the proportion of cases cured between 2009 and 2015.

Keywords: tuberculosis; epidemiology; geographic distribution; historic serie.

\section{INTRODUÇÃO}

A tuberculose (TB) - caracterizada pela Organização Mundial de Saúde (OMS) como emergência sanitária mundial - é uma doença de impacto global. Nos últimos cinco anos, constituise a nona principal causa de morte e a principal causa de morte por um único agente infeccioso no mundo'. Seu controle está previsto pela Organização das Nações Unidas (ONU), que estipulou uma incidência de TB ativa menor que 10/100 mil habitantes até 2035 1,2.

O complexo Mycobacterium tuberculosis compreende um grupo composto por bactérias que tipicamente afetam os pulmões (TB pulmonar), mas também podem afetar outros tecidos e órgãos (TB extrapulmonar) ${ }^{1}$.

Apesar de estar em declínio em áreas da Europa, Pacífico Ocidental e Sudeste da Ásia, a TB está fora de controle em regiões como Sudeste da África, Irã e Índia². A OMS estimou cerca de 10,4 milhões de casos de TB no mundo em 2016, com aproximadamente 1,3 milhão de mortes causadas pela doença, superando os óbitos relacionados ao HIV1,3.

Entre os 30 países com maior incidência de TB e que, juntos, foram responsáveis por aproximadamente $87 \%$ dos casos em todo o mundo em 2016, o Brasil ocupava a 20a posição ${ }^{1,4}$. Em 2015, registraram-se 63,7\% casos de coinfecção HIV-TB dentre os óbitos associados à TB em território nacional ${ }^{5}$. Quanto aos 4.610 óbitos por TB registrados nessa época, 76,7\% foram atribuídos à forma pulmonar, e cerca de 44\% das mortes concentraram-se na região Sudeste, na qual o estado de Minas Gerais (MG) ocupava o terceiro lugar das piores taxas de cura, variando de $72 \%$ a $71 \%$ de 2004 a $2015^{6}$.

Nesse contexto, encontra-se o município de Ouro Preto (OP), MG, que apresenta relação histórica íntima com a TB desde o princípio da exploração mineradora. Os primeiros óbitos por TB relacionados à mineração foram registrados em Passagem de Mariana (região de exploração da companhia The Ouro Preto Gold Mines) entre 1891 e 1898, sendo a incidência da doença crescente desde essa época. A partir de então, caracterizou-se toda a região como endêmica para TB (à época denominada "doença de mina"), com algum sucesso das medidas sanitárias para redução das taxas de incidência apenas a partir de $1927^{7}$.

De acordo o censo de 2010 do IBGE, OP apresenta uma população total de 70.281 habitantes, dos quais 40.916 habitam a sede municipal ${ }^{8}$. Possui 12 distritos com população variável entre 403 e 8.923 habitantes, e média de 2.447 habitantes/distrito. Além da atividade mineradora, ressalta-se o artesanato em pedra-sabão entre as principais atividades econômicas distritais ${ }^{9}$. Considerando-se a relação histórica deste município com a TB e as particularidades do processo de urbanização e exposição a poeiras minerais em OP e seus distritos, este estudo buscou investigar uma série histórica de casos de TB em um período de 17 anos, em associação com sua distribuição temporal e geográfica.

\section{MÉTODO}

\section{Delineamento do estudo}

Estudo retrospectivo descritivo e de séries temporais entre 1999 e 2015, baseado em dados secundários de sistema próprio de registro de TB da Secretaria Municipal de Saúde (SMS) de OP. Trata-se de uma base de dados com atualização mensal sob responsabilidade da vigilância epidemiológica do município, implementada há mais de duas décadas, por iniciativa municipal, para registro de casos e formulação de estratégias de saúde pública. A série temporal analisada representou o maior recorte de tempo com disponibilidade de dados no Sistema de Informação de Agravos de Notificação (SINAN) e no sistema de registro municipal. 


\section{Casuística e coleta de dados}

Os dados necessários ao estudo foram obtidos no sistema próprio de registros de TB do município e por meio de busca ativa em prontuários médicos. Ao todo, foram computados 305 registros de TB, com exclusão de três duplicados e dois incorretos. Foram mantidos quatro registros com endereços extramunicipais, mas tratados em OP. Ao final, obtiveram-se 300 registros de TB no período em estudo.

O número de registros constantes na SMS foi comparado à quantidade de casos notificados no SINAN para verificação da abrangência da cobertura do sistema municipal de registros.

\section{Análises estatísticas}

Para minimizar erros provenientes da flutuação populacional, o cálculo das incidências de TB foi realizado pela divisão do número de casos pela população estimada pelos censos de 2000 e 2010 e projeções intercensitária para os demais anos ${ }^{10}$. Quando analisadas populações masculina ou feminina, foi considerada a média desses extratos populacionais dada pelos censos de 2000 e $2010^{8,10}$. A proporção de pacientes curados foi dada pela razão entre casos com evolução para cura e total de pacientes com TB confirmada. As variáveis contínuas foram apresentadas como média \pm desvio-padrão. Variáveis discretas ou não paramétricas foram apresentadas como mediana e distância interquartílica (DIQ).

Para as análises de tendência, foram utilizados modelos de regressão polinomial para séries históricas. Foram consideradas como variáveis dependentes $(Y)$ as taxas de incidência de tuberculose e proporção de pacientes curados. Como variável independente $(X)$, considerou-se o período de 1999 a 2015 para incidência de TB e 1999 a 2014 para proporção de pacientes curados. O ano 2015 foi excluído da série temporal da proporção de pacientes curados devido ao desconhecimento do desfecho dos casos ainda em tratamento até o fechamento do estudo. Para evitar correlação serial entre os termos da equação de regressão, foi realizada transformação da variável independente "período" em "período-centralizado", dado pelo ano menos ano médio da série histórica, conforme sugerem Latorre e Cardoso" ${ }^{11}$.

A modelagem das séries históricas deu-se pela plotagem de diagramas de dispersão e avaliação dos melhores modelos de ajuste de curva de regressão. Optou-se pela construção de modelos com complexidade gradual, iniciando-se pelo modelo linear simples, seguindo-se por polinômios de até sétima ordem e modelo não linear do tipo exponencial. Para escolha do modelo mais adequado, considerou-se o coeficiente de determinação $\left(R^{2}\right)$, ganho em $R^{2}$ em relação ao modelo anterior e significância. Quando dois modelos apresentaram resultados semelhantes, optou-se por aquele de menor complexidade. Associação entre variáveis foi verificada através do método do Qui-quadrado $\left(x^{2}\right)$ ou exato de Fischer. Comparação de médias foi dada pelo teste $T$ de Student e de medianas pelo $U$ de Mann-Whitney quando os pressupostos de normalidade não foram atendidos. Medidas de tamanho de efeito foram dadas pela razão de chances (odds ratio) quando utilizado o Qui-quadrado, ou $d$ de Cohen quando realizado teste $T$ de Student. O cálculo do $d$ de Cohen é dado pelas seguintes equações ${ }^{12}$ :

$$
d=\frac{M 1-M 2}{\text { Scombinado }} \quad \text { Scombinado }=\sqrt{\frac{\left(n_{1}-1\right) s_{1}^{2}+\left(n_{2}-1\right) s_{2}^{2}}{n_{1}+n_{2}-2}}
$$

Onde $\mathrm{M} 1$ = média do primeiro grupo, $\mathrm{M} 2$ = média do segundo grupo, $\mathrm{n} 1$ = tamanho amostral do primeiro grupo, $\mathrm{n} 2$ = tamanho amostral do segundo grupo, $\mathrm{s} 1$ = desvio-padrão do primeiro grupo e s2 = desvio-padrão do segundo grupo.

As análises estatísticas foram realizadas no software SPSS Statistics 17.0, assumindo-se valor crítico de $p$ igual a $5 \%$ para a rejeição das hipóteses nulas.

\section{Considerações éticas}

O projeto de pesquisa foi aprovado pelo Comitê de Ética em Pesquisa da UFOP (CAAE: 55149516.3.0000.5150) e nenhum dos autores apresentam conflitos de interesses. 


\section{RESULTADOS}

Dos 300 registros atribuídos à TB no período em estudo, 293 mantiveram-se sem mudança de diagnóstico após consultas aos prontuários. Maior proporção de casos foi observada no sexo masculino, com média de 35,20 casos/100 mil homens e 15,75 casos/100 mil mulheres, representando uma taxa 2,23 vezes maior entre homens. A idade média foi de 40,3 $\pm 16,4$ anos, incluindo pacientes desde o primeiro até o $92^{\circ}$ ano de vida. A forma de TB mais observada foi a pulmonar, com 246 pacientes ( $84,0 \%)$, seguida da pleural $(6,8 \%)$ e ganglionar $(3,7 \%)$ (Tabela 1$)$. Observaram-se ainda um caso de tuberculose renal, dois casos de tuberculose testicular e um caso de tuberculose peritoneal.

Foi constatada sorologia para HIV em apenas $16,3 \%$ dos 300 registros. Entre os pacientes triados, 20,4\% (10 casos) foram sororreagentes e 79,6\% (39 casos) não reagentes. O percentual de sorologia entre 2001 e 2015 foi de 19,8\%, sendo 7,7\% e 22,2\% correspondentes ao início e fim deste extrato temporal (dados incluídos para comparação com o SINAN). Para os casos de TB pulmonar, pesquisa de BAAR foi realizada em 77,2\% dos pacientes (Tabela 1), com 59,1\% com resultado positivo.

O principal desfecho observado foi a cura, que ocorreu em 70,0\% dos casos (desfecho positivo). Desfechos negativos totalizaram $20,2 \%$ dos registros, dos quais $10,6 \%$ foram referentes a óbitos e $9,6 \%$ a abandono de tratamento. A relação de indivíduos com desfecho desconhecido totalizou 14 casos $(4,8 \%)$, e 5,0\% dos pacientes foram transferidos para unidades de saúde extramunicipais, tendo também desfecho desconhecido para a SMS-OP.

Homens apresentaram chance (odds ratio) 2,07 vezes maior, quando comparados às mulheres, para desfechos negativos, com intervalo de confiança a $95 \%$ de 1,03 a 4,16 (Qui-quadrado, $p=0,037$ ). A idade média foi significativamente maior no grupo com desfecho negativo quando comparado ao grupo com desfecho positivo, com resultado de 49,38 $\pm 14,81 \mathrm{e}$ $37,47 \pm 15,92$ anos, respectivamente ( $T$ de Student $p<0,001$ ), com tamanho de efeito moderado $(d \text { de Cohen }=0,76)^{11,12}$.

Em relação à distribuição geográfica dos casos, verificou-se índice sede/distrital de 2,56. A taxa de incidência média foi de 29,76 e 16,23 casos/100 mil habitantes na sede municipal e na zona distrital, respectivamente (Tabela 2). Não foi observada associação entre zona de habitação (sede ou distritos) e tipo de desfecho - positivo ou negativo - (Qui-quadrado, $\mathrm{p}=0,434$ ).

Tabela 1. Dados descritivos da tuberculose entre 1999 e 2015. Ouro Preto, MG.

\begin{tabular}{ccccccc} 
& Pulmonar & Pleural & Ganglionar & $\begin{array}{c}\text { Outras } \\
\text { Apresentações* }\end{array}$ & Desconhecido** & Total \\
\hline Casos (\%) & 84,0 & 6,8 & 3,7 & 3,1 & 2,4 & 100 \\
\hline $\mathbf{N}$ & 246 & 20 & 11 & 9 & 7 & 293 \\
\hline Índice H/M & 2,11 & 4,0 & 1,75 & 2,0 & 1,3 & 2,15 \\
\hline N & 246 & 20 & 11 & 9 & 7 & 293 \\
\hline Idade & $40,2 \pm 16,0$ & $40,4 \pm 15,3$ & $32,5 \pm 17,4$ & $50,1 \pm 19,1$ & $41,0 \pm 27,3$ & $40,3 \pm 16,4$ \\
\hline N & 231 & 19 & 11 & 9 & 5 & 275 \\
\hline Baciloscopia & 77,2 & 30,0 & 0 & 0 & 0 & 66,9 \\
\hline N & 190 & 6 & 11 & 9 & 7 & 196 \\
\hline Cura (\%) & 70,3 & 80,0 & 81,8 & 55,6 & 28,6 & 70,0 \\
\hline N & 246 & 19 & 11 & 7 & 7 & 293 \\
\hline
\end{tabular}

$\mathrm{N}=293$ (casos sem mudança de diagnóstico). Índice $\mathrm{H} / \mathrm{M}=$ casos masculinos/femininos. *Casos registrados como tuberculose testicular, peritoneal, renal ou extrapulmonar não discriminada. ** apresentação não

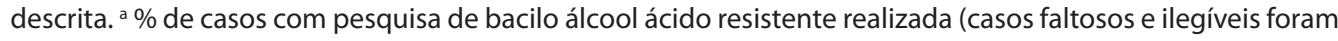
computados como não realizados). ${ }^{\mathrm{b}} \%$ de cura dos casos sem mudança de diagnóstico.

Fonte: Registros da Secretaria Municipal de Saúde de Ouro Preto, MG. 
Tabela 2. Distribuição de novos casos de tuberculose segundo localidade entre os anos de 1999 e 2015 . Ouro Preto, MG.

\begin{tabular}{|c|c|c|c|}
\hline Localidade & Novos casos & População & Incidência Média \\
\hline Amarantina & 12 & 3577 & 19,73 \\
\hline Antônio Pereira & 10 & 4480 & 13,13 \\
\hline Cachoeira do Campo & 12 & 8923 & 7,91 \\
\hline Engenheiro Correa & 0 & 403 & 0,00 \\
\hline Glaura & 2 & 1418 & 8,30 \\
\hline Lavras Novas & 3 & 929 & 19,00 \\
\hline Miguel Burnier & 3 & 809 & 21,81 \\
\hline Rodrigo Silva & 13 & 1080 & 70,81 \\
\hline Santa Rita de O. Preto & 14 & 4243 & 19,41 \\
\hline Santo Antônio do Leite & 9 & 1705 & 31,05 \\
\hline Santo Antônio do Salto & 2 & 1068 & 11,02 \\
\hline São Bartolomeu & 1 & 730 & 8,06 \\
\hline Sede & 207 & 40.916 & 29,76 \\
\hline Total Distrital & 81 & 29.365 & 16,23 \\
\hline Índice S/D & 2,56 & & \\
\hline
\end{tabular}

$\mathrm{N}=288$. O local de residência de 5 indivíduos é desconhecido ou extramunicipal. Incidência média do período em estudo dada como casos/100 mil habitantes. Índice S/D = casos na sede municipal/casos distritais.

Fonte: Registros da Secretaria Municipal de Saúde de Ouro Preto, MG. Populações segundo censo de 2010 do IBGE

A aglomeração domiciliar foi avaliada indiretamente pelo número de contatos domiciliares registrados e não constituiu diferença significativa entre sede ou distritos, com mediana de quatro e DIQ de quatro contatos em ambas regiões (Mann-Whitney $p=0,544$ ). As diferenças na aglomeração domiciliar segundo desfecho também não se mostraram significativas (MannWhitney $p=0,278$.

A incidência média da TB no período foi de $22,9 \pm 2,5$ novos casos $/ 100$ mil habitantes, com variação de 40,74 casos/100 mil habitantes em 2000 (maior taxa do período) até 8,10 casos/100 mil habitantes em 2015 (menor taxa do período) (Tabela 3). A análise da série temporal revela queda na taxa de incidência da TB ao longo dos anos, considerando-se como melhor ajuste de curva um modelo de regressão exponencial com $R^{2}$ de $80,6 \%(p<0,001)$ (Figura 1).

Entretanto, quando avaliada a proporção de casos curados em relação aos demais desfechos, elegeu-se como melhor modelo regressivo ajustado um polinômio de sexta ordem com $\mathrm{R}^{2}$ de $88,0 \%(p=0,001)$. Essa série temporal sugere aumento na proporção de casos curados entre 1999 e 2005, com queda entre 2009 e o fim da série. Entre os anos de 2005 e 2009, observou-se comportamento atípico, com queda (atingindo-se os menores valores desse recorte temporal em 2007), seguida por ascensão a valores próximos aos observados no início do recorte em 2009 (Figura 1). Esse período atípico constitui possível explicação para o não ajuste do modelo a uma curva quadrática (modelo linear de segunda ordem) para a qual se observou o maior ganho de $\mathrm{R}^{2}$ em relação ao modelo imediatamente menos complexo $(57,3 \%)$. As informações sobre os modelos construídos para ambas as séries temporais encontram-se na Tabela 4.

Dados recuperados do SINAN apontam 181 casos confirmados e notificados de TB em OP entre 2001 e 2015 (informações para 1999 e 2000 não se encontram disponíveis para consulta eletrônica). No entanto, os registros da SMS informam que o município notificou ao 
Tabela 3. Taxas de incidência e proporção de cura de tuberculose entre os anos de 1999 e 2015. Ouro Preto, MG.

\begin{tabular}{|c|c|c|}
\hline Ano & Incidência & Proporção de pacientes curados \\
\hline 1999 & 39,29 & $59,26 \%$ \\
\hline 2000 & 40,74 & $65,52 \%$ \\
\hline 2001 & 32,98 & $65,38 \%$ \\
\hline 2002 & 19,38 & $75,00 \%$ \\
\hline 2003 & 34,11 & $86,96 \%$ \\
\hline 2004 & 26,39 & $88,89 \%$ \\
\hline 2005 & 34,97 & $88,89 \%$ \\
\hline 2006 & 18,82 & $73,33 \%$ \\
\hline 2007 & 26,85 & $65,00 \%$ \\
\hline 2008 & 20,22 & $68,75 \%$ \\
\hline 2009 & 14,39 & $83,33 \%$ \\
\hline 2010 & 21,34 & $77,78 \%$ \\
\hline 2011 & 15,58 & $75,00 \%$ \\
\hline 2012 & 11,29 & $62,50 \%$ \\
\hline 2013 & 12,27 & $55,56 \%$ \\
\hline 2014 & 12,21 & $33,33 \%$ \\
\hline 2015 & 8,10 & * \\
\hline
\end{tabular}

*O ano de 2015 foi excluído do cálculo de proporção de pacientes curados por não se ter a informação sobre o desfecho de todos os casos em tratamento até o fechamento do estudo. $\mathrm{N}=293$ (casos sem mudança de diagnóstico). Incidências apresentadas como casos/100mil habitantes.

SINAN 244 casos no mesmo período, totalizando diferença de 63 eventos $(25,82 \%)$ - perda de informação. Os livros de registros da SMS-OP, que possui seu próprio método de contagem de casos notificados e não notificados, apontam para 52 eventos efetivamente não notificados pelo município ao SINAN dentre os 300 registros da série em estudo - subnotificação. Assim, observou-se tendência marginalmente não significante (Qui-quadrado $p=0,052$ ) na associação entre desfechos negativos e subnotificação dos casos de TB avaliados pelo banco de dados da SMS-OP, indicando chance (odds ratio) 1,99 vezes maior de desfechos negativos entre casos não notificados quando comparados aos notificados. No entanto, o intervalo de confiança limita as conclusões possíveis dessa associação (IC 95\% 0,98 a 4,01).

\section{DISCUSSÃO}

Segundo o MS (2017), a incidência de casos de TB no Brasil apresentou queda de 51,4 para 33,7 casos/100 mil habitantes entre os anos de 1999 e 2015, enquanto MG apresentou queda de 35,4 para 16,2 casos/100 mil habitantes neste mesmo período. De forma análoga, nesta série histórica observou-se queda na incidência, porém de forma mais acentuada que aquela observada no estado (39,3 para 8,10 casos/100 mil habitantes), com incidência $50 \%$ menor que a observada em MG ao fim da série histórica. Para a incidência média, o país apresentou valores de 40,3; MG, 22,7 e OP, 22,9 casos/100 mil habitantes. Observou-se, assim, que a taxa de incidência média de OP aproximou-se à do estado de MG e manteve-se distante daquelas observadas para o país ${ }^{13}$.

As proporções observadas de TB entre os sexos concordam com os boletins epidemiológicos de MG, em que foram constatados valores de 23,8 e 10,7 casos/100 mil habitantes para 

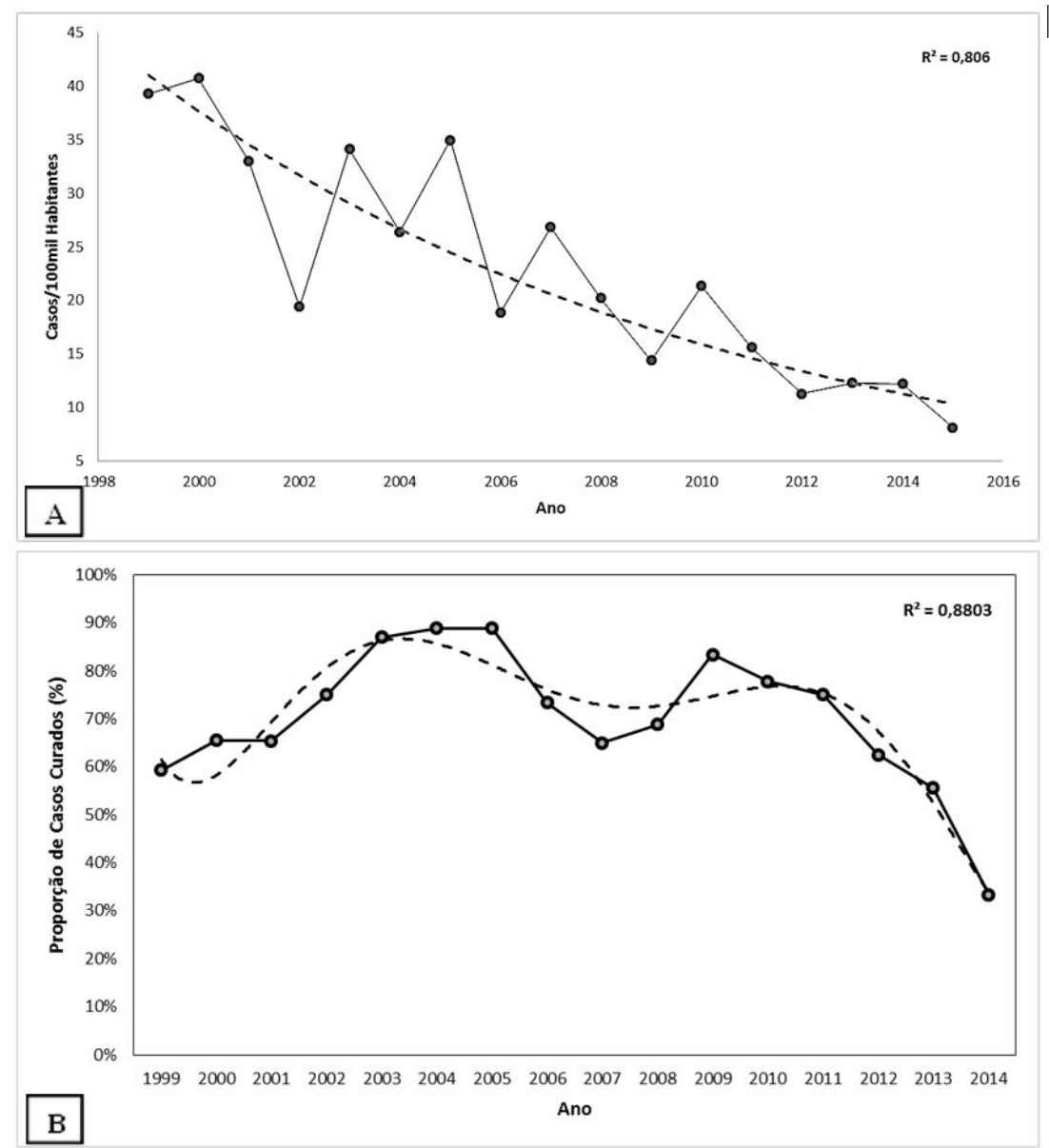

Figura 1. Série temporal da taxa de incidência e casos curados de tuberculose em Ouro Preto, MG. A: N = 293. Os parâmetros do modelo foram: constante 20,59, b1 -0,086, $\mathrm{p}$ (modelo) $<0,001$. A incidência anual é apresentada como casos/100 mil habitantes. A linha tracejada representa o modelo de regressão. A variação nas taxas de incidência ao longo do período é representada pela linha contínua. $\mathbf{B}: \mathrm{N}=285$. Os parâmetros definidos para o modelo foram: Constante 76,07, b1 -4,510, b2 1,012, b3 0,335, b4 -0,065, b5 -0,005, b6 0,001, $p$ (modelo $)=0,001$. A proporção de casos curados em um período é dada pela razão dos casos com evolução para cura sobre o total de casos confirmados para este período. O ano de 2015 foi excluído da série temporal por não se conhecer o desfecho de todos os casos em tratamento neste ano até o fechamento do estudo

homens e mulheres, respectivamente, em 2014, o que representa uma taxa 2,2 vezes maior para o sexo masculino. Proporção semelhante é observada nacionalmente, com 46,2 e 22,3 casos/100 mil habitantes para homens e mulheres, respectivamente (taxa 2,07 vezes maior para o sexo masculino $)^{14}$. Em OP, foi ainda observada maior incidência na sede municipal em relação aos distritos, fato esperado, dado o mecanismo de transmissão da doença

A tuberculose (TB) ativa em pessoas que vivem com HIV/AIDS é a condição de maior impacto na mortalidade por AIDS e por TB no país. Dentre as intervenções preconizadas pelo MS para controle da coinfecção TB-HIV, destaca-se a testagem oportuna para HIV para todos os portadores de $\mathrm{TB}^{15}$. Contudo, a realização de sorologia para HIV em OP foi de apenas 19,8\% dos casos entre 2001 e 2015, e contrasta com os valores apresentados por MG, com sorologia realizada em $49,8 \%$ dos casos observados no mesmo período. Observa-se que essa proporção tende a aumentar com a progressão da série histórica em MG, sendo o percentual de triagem para HIV de 31,9\% em 2001 e 76,5\% em 2015. Embora OP acompanhe essa tendência, os dados apresentados revelam que o município ainda apresenta notória fragilidade na investigação da coinfecção HIV-TB, quando comparada ao progresso apresentado pelo estado. Ressalta-se que a presença de HIV como fator de risco para TB está mais associada ao desenvolvimento de cepas multirresistentes, aliada a fatores como alcoolismo e tabagismo ${ }^{16,17}$. 


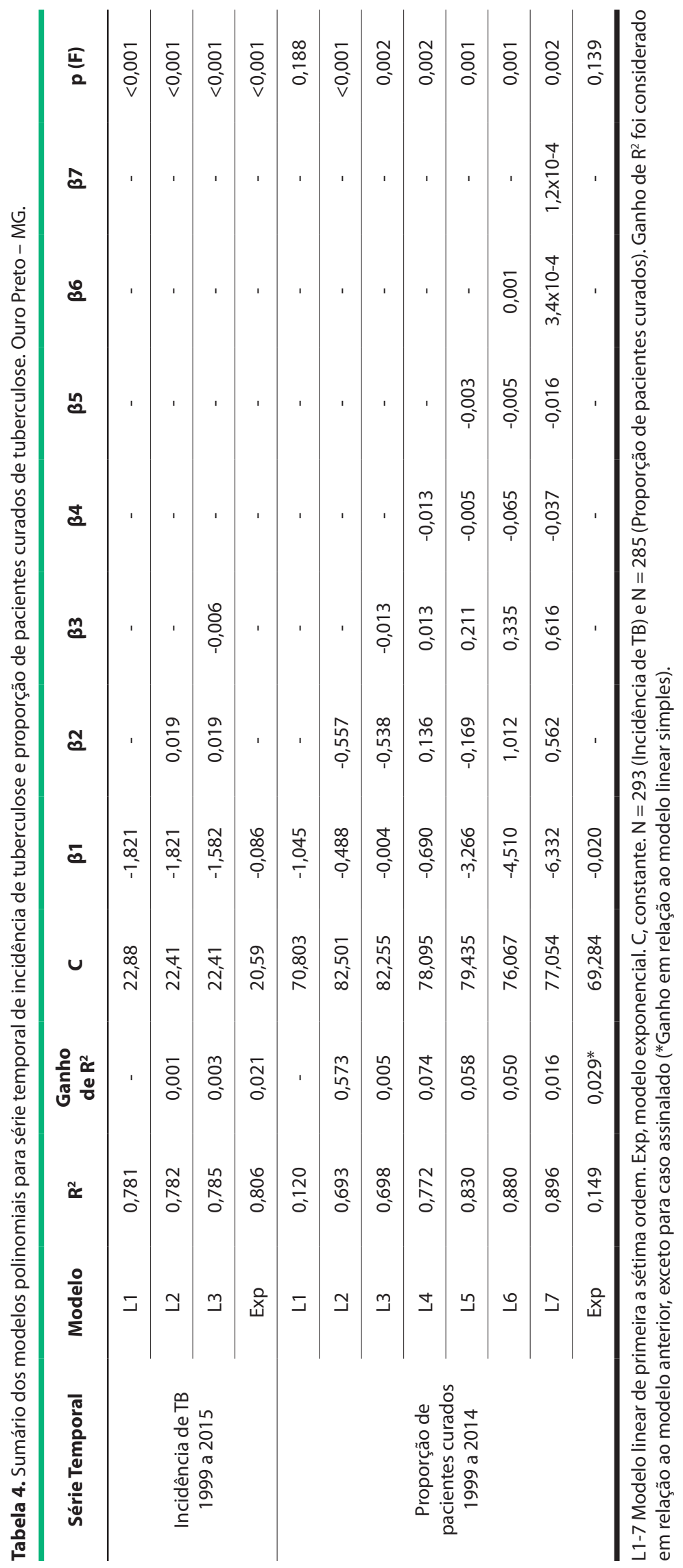


A forma clínica predominante observada foi a pulmonar, que consiste na apresentação mais comum da doença e forma de maior interesse epidemiológico devido à sua transmissibilidade ${ }^{14,18,19}$. Apesar de o MS recomendar a coleta de duas a três amostras de escarro espontâneo para pesquisa direta de BAAR em todos os pacientes sintomáticos respiratórios ${ }^{20}$, em OP essa pesquisa foi realizada em apenas $77,2 \%$ dos pacientes, dos quais $59,1 \%$ apresentaram resultado positivo. A média de confirmação bacteriológica laboratorial por meio de pesquisa direta, cultura ou teste rápido molecular, por sua vez, foi de $72,6 \% \mathrm{em}$ MG e $71,6 \%$ no Brasil, em $2016^{6}$.

A tendência de queda na incidência de TB em OP ao longo dos anos está em consonância com dados nacionais apresentados pelo $\mathrm{MS}^{19}$. Embora o boletim epidemiológico do MS não apresente o melhor ajuste de curva a um modelo regressivo, a plotagem dos dados apresentada não sugere queda exponencial nas taxas de incidência, como observadas para OP. Contudo, a análise isolada desse achado é insuficiente para garantir a efetividade das ações em saúde no combate à TB no município, especialmente ao considerar os pontos de fragilidade já apresentados.

Outro dado concordante com os boletins epidemiológicos foi a associação entre desfechos negativos e idade maior que 60 anos ${ }^{5,14}$. Os resultados da série em estudo revelam uma maior tendência a desfechos negativos em média populacional próxima à descrita pela literatura. Não menos importante, o amplo acometimento da população urbana em idade produtiva, ainda que com uma maior tendência a desfechos positivos, destaca o comprometimento da força de trabalho dos conglomerados urbanos.

A taxa de cura de TB em OP, nos últimos anos, foi marcada por oscilações que culminaram com declínio a partir de 2009 para um valor final da série, em 2014, de 33,3\%. Tal resultado, nesse mesmo ano, correspondeu a menos da metade da taxa de cura em MG $(76,2 \%)$ e da média nacional $(74,2 \%)^{21}$. Comparativamente, todos revelaram-se abaixo das taxas de países como a Tailândia (80\%) e a Indonésia (84\%), cujos valores se aproximaram ao do município de OP $(83,3 \%)$ no início do período em estudo (2009) ${ }^{22}$. Essa queda de aproximadamente $50 \%$ nas taxas de cura da TB em OP em intervalo de apenas cinco anos poderia ser explicada por três aspectos principais: falha na ingestão medicamentosa, dificuldade de acesso ao sistema de saúde ou descontinuidade do tratamento. A descontinuidade do tratamento como explicação para esse fenômeno é improvável, dado que a taxa de abandono observada neste estudo foi menor $(9,3 \%)$ que as apresentadas pelo estado de MG (11,5\%) e pelo Brasil (11\%) em $2014^{21}$.

A falha na ingestão medicamentosa pode ser evitada pelo tratamento diretamente observado (TDO), com supervisão da tomada dos fármacos antituberculose por profissional de saúde ${ }^{1,23}$. Entretanto, os registros consultados não discriminavam para quais casos confirmados a estratégia do TDO foi adotada, o que limita as conclusões deste estudo sob esse prisma. A dificuldade de acesso é um ponto provável, posto que OP possui aproximadamente 13,0\% da população pertencente à região rural e distrital, cobrindo área geograficamente extensa.

Assim, embora se tenha observado queda substancial na taxa de incidência da doença, a proporção de pacientes curados não acompanha essa redução no número de novos casos, ponto que retoma a possibilidade de superestimação da redução de casos por subnotificação, especialmente nas regiões mais vulneráveis do município. Ainda no que concerne ao desfecho da doença, verificou-se percentual de óbitos superior à média observada para MG e para o Brasil entre 2005 e 2014, com valores de aproximadamente $6,4 \%$ contra $10,3 \%$ observado neste estudo ${ }^{14}$.

Portanto, apesar da relação histórica entre mineração e TB no município, observa-se que OP ainda apresenta preocupantes vulnerabilidades no que tange à notificação, registro de casos, desfechos e taxas de cura. Além dos dispostos, especial atenção deve ser dada à relação entre exposição ocupacional a poeiras minerais e a TB, sobretudo em alguns distritos. A vigilância dos ambientes de trabalho deve ser priorizada a fim de eliminar ou reduzir ao máximo a emissão de particulados e outros fatores de risco 24,25 .

Também se faz necessária a melhoria da vigilância epidemiológica da doença, incluindo o sistema oficial de notificações, e das ações voltadas para o cuidado dos pacientes acometidos. 


\section{REFERÊNCIAS}

1. World Health Organization. Global tuberculosis report 2017. Genevra: WHO; 2017.

2. Pai M, Behr MA, Dowdy D, Dheda K, Divangahi M, Boehme CC, et al. Tuberculosis. Nat Rev Dis Primers. 2016;27(2):16076. http://dx.doi.org/10.1038/nrdp.2016.76. PMid:27784885.

3. Khan MS, Fletcher H, Coker R, London School of Hygiene and Tropical Medicine TB Centre Steering Committee, Coker R. Investments in tuberculosis research - What are the gaps? BMC Med. 2016;14(1):123. http://dx.doi.org/10.1186/s12916-016-0644-0. PMid:27558175.

4. Brasil. Ministério da Saúde. Secretaria de Vigilância em Saúde. Boletim Epidemiológico - Coinfecção TBHIV no Brasil: panorama epidemiológico e atividades colaborativas. Brasília: Ministério da Saúde; 2017.

5. Brasil. Ministério da Saúde. Programa nacional de controle da tuberculose. Brasília: Ministério da Saúde; 2017.

6. Brasil. Ministério da Saúde. Secretaria de Vigilância em Saúde. Boletim Epidemiológico - Indicadores prioritários para o monitoramento do Plano Nacional pelo Fim da Tuberculose como Problema de Saúde Pública no Brasil. Brasília: Ministério da Saúde; 2017.

7. Souza RF, Machado MHPT. Trabalho e cotidiano na mineração aurífera inglesa em Minas Gerais: a mina da Passagem de Mariana (1863-1927) [thesis]. São Paulo: Universidade de São Paulo; 2009.

8. Instituto Brasileiro de Geografia e Estatística. Censo 2010. Brasília: IBGE; 2010.

9. Carvalho CG, Silva JM, Curi A, Flores JCC. A dependência da arrecadação do município de Ouro Preto do setor mineral. R Esc Minas. 2012;65(3):385-92. http://dx.doi.org/10.1590/S0370-44672012000300016.

10. Brasil. Ministério do Planejamento, Desenvolvimento e Gestão. Instituto Brasileiro de Geografia e Estatística. Censos e Projeções Intercensitárias - 1999 a 2015. Brasília: IBGE.

11. Latorre MRDO, Cardoso MRA. Análise de séries temporais em epidemiologia: uma introdução sobre os aspectos metodológicos. Rev Bras Epidemiol. 2001;4(3):145-52. http://dx.doi.org/10.1590/S1415$790 \times 2001000300002$.

12. Cohen J, Hillsdale NJ. Statistical power analysis for the behavioral sciences. Hillsdale, NJ: Lawrence Erlbaum Associates; 1988.

13. Brasil. Ministério da Saúde. Série histórica do coeficiente de incidência de tuberculose. Brasil, regiões e unidades federadas de residência por ano de diagnóstico (1990 - 2016) [Internet]. [citado em 2019 mar 30]. Disponível em: http://portalarquivos2.saude.gov.br/images/pdf/2017/novembro/22/taxa-incidenciatuberculose-1990-2016-MAl-2017.pdf

14. Brasil. Ministério da Saúde. Panorama da tuberculose no Brasil: a mortalidade em números. Brasília: Ministério da Saúde; 2016.

15. Brasil. Ministério da Saúde. Recomendações para o manejo da coinfecção TB-HIV em serviços de atenção especializada a pessoas vivendo com HIV/AIDS. Brasília: Ministério da Saúde; 2013.

16. Dheda K, Gumbo T, Gandhi NR, Murray M, Theron G, Udwadia Z, et al. Global control of tuberculosis: from extensively drug-resistant to untreatable tuberculosis. Lancet Respir Med. 2014;2(4):321-38 PMid:24717628.

17. Carvalho CN, Dourado I, Bierrenbach AL. Subnotificação da comorbidade tuberculose e aids: uma aplicação do método de linkage. Rev Saude Publica. 2011;45(3):548-55. http://dx.doi.org/10.1590/S003489102011005000021 . PMid:21503555.

18. Maciel ELN, Sales CMM. A vigilância epidemiológica da tuberculose no Brasil: como é possível avançar mais? Epidemiol Serv Saude. 2016;25(1):175-8. PMid:27861690.

19. Brasil. Ministério da Saúde. Secretaria de Vigilância em Saúde. Boletim Epidemiológico - Detectar, tratar e curar: desafios e estratégias brasileiras frente à tuberculose. Brasília: Ministério da Saúde; 2015; 46(9).

20. Fundação Nacional de Saúde. Tuberculose: guia de vigilância epidemiológica. Brasília: FUNASA; 2002.

21. Brasil. Ministério da Saúde. Secretaria de Vigilância em Saúde. Boletim Epidemiológico - Perspectivas brasileiras para o fim da tuberculose como problema de saúde pública. Brasília: Ministério da Saúde; 2016. Vol. 47, No. 13

22. World Health Organization. Global tuberculosis report 2016. Genevra:WHO; 2016.

23. Ministério da Saúde. Brasil livre da tuberculose: plano nacional pelo fim da tuberculose como problema de saúde pública. Brasília: Ministério da Saúde; 2017.

24. teWaterNaude JM, Ehrlich RI, Churchyard, GJ, Pemba L, Dekker K, Vermeis M, White NW, Thompson ML, Myers JE. Tuberculosis and sílica exposure in South African gold miners. Occup Environ Med. 2006;63(03):187-92.

25. Barboza CEG, Winter DH, Seiscento M, Santos UP, Terra Filho M. Tuberculose e silicose: epidemiologia diagnóstico e quimioprofilaxia. J Bras Pneumol. 2008;34(11):959-66. http://dx.doi.org/10.1590/S180637132008001100012 . PMid:19099104. 